PREHOSPITAL CARE

\title{
Use of a forensic technique to identify blood contamination of emergency department and ambulance trauma equipment
}

\author{
J B Lee, M Levy, A Walker
}

Using a Kastle-Meyer (KM) technique, the following equipment from the emergency departments of six UK hospitals (four trusts) and three regional ambulance services was tested for blood contamination: extrication ("spinal") boards, cervical collars, straps, box splints, head blocks, and headboards. Only equipment ready for patient use was tested. Over half of trauma equipment $(57 \%)$ tested positive for blood, including $15 \%$ of equipment that was visibly stained with blood. There have been no recorded cases of infection from contaminated trauma equipment but our study has identified the potential risk. Disposable covers for boards, disposable straps, and disposable radiolucent head blocks which are currently available provide a solution but have resource implications

$\mathrm{F}$ orensic techniques have been used to identify blood contamination of dental, ${ }^{1}$ radiological, ${ }^{2}$ and anaesthetic ${ }^{3}$ equipment that appeared clean to the naked eye. The authors of these studies considered that the contaminated surfaces presented a greater risk of potential infection transmission to medical and dental staff than to patients. Equipment used to transport trauma patients may come into direct contact with open wounds. We used a forensic test to determine the extent of blood contamination of trauma equipment in a sample of ambulances and emergency departments in the UK to establish whether a potential risk of infection transmission between trauma patients exists.

\section{METHODS}

Six UK Emergency Departments (four hospital trusts) and three regional ambulance services were included in the study. Approval for testing was granted by the lead clinician of each department and the operational or medical director of the individual ambulance services with agreement that their centres would remain individually anonymous. Ethical approval was sought but not required.

Using a Kastle-Meyer (KM) technique, the following trauma equipment was tested over two weeks in 2004: extrication ("spinal") boards, cervical collars, straps, box splints, head blocks, and head boards. A validation test was first performed to exclude false negative results by confirming the ability of the reagent to identify (horse) blood. The area under investigation was then swabbed with a cotton bud, a drop of Kastle-Meyer solution added, followed by a drop of hydrogen peroxide. A colour change to pink was taken as an indication of the presence of blood. The testing kit supplied by Scenesafe (The Forensic Science Service, Chorley, UK) contained all of the above components and is identical to that used by the Metropolitan Police for identification of blood at crime scenes.
Convenience sampling of equipment in emergency departments, ambulances arriving with non-trauma patients, and ambulance stations was performed. Only equipment ready for patient use was tested. Individual emergency department staff and ambulance crews were not warned of our visits. Standardised areas, considered by the authors as those most likely to come into contact with the patient were swabbed, as shown in the following list:

- medial side of head blocks

- inner side of head straps

- patient side of head boards

- patient side of straps and buckles

- patient side of extrication boards (head, feet, and hand regions)

- patient side of box splints

- back and chin area of foam in cervical collars.

A maximum of four areas (about $3 \mathrm{~cm}$ square in size) were tested for each piece of equipment unless the equipment appeared visibly blood stained, in which event the stain was tested to confirm that it was blood. Testing was performed in pairs by the authors. The result was independently recorded as positive or negative by the two observers to determine interobserver agreement. In the event of disagreement, retesting of the same area was undertaken immediately.

The outcome measures of interest were: (1) equipment visibly or not visibly stained with blood and (2) positive or negative result for blood on Kastle-Meyer testing.

Equipment was considered contaminated if any area of it tested positive for blood. We decided a priori to perform a subset analysis on "hospital cleaned" versus "ambulance crew cleaned" trauma equipment with respect to blood contamination. A z-test was performed to calculate confidence intervals around the difference in proportions of contamination between the two groups.

\section{RESULTS}

Fifteen per cent of equipment was visibly contaminated with blood. A further $42 \%$ of equipment, not visibly contaminated, tested positive for blood with the Kastle-Meyer test. Observers agreed on colour change on all samples tested. See tables 1 and 2 for comparative results.

\section{DISCUSSION}

The Kastle-Meyer test ${ }^{4}$ is a presumptive test for haemoglobin which relies on the peroxidase-like activity of the haem molecule and uses phenolphthalein as a colour indicator (see fig 1). It is very specific for blood and although not as sensitive as other reagents can still identify blood at 1 part per

Abbreviation: DoH, Department of Health 
Table 1 Overall blood contamination on Kastle-Meyer testing by equipment type

\begin{tabular}{lc}
\hline Equipment & Proportion testing +ve for blood \\
\hline Extrication boards & $26 / 36(72 \%)$ \\
Boxsplints & $36 / 52(69 \%)$ \\
Head blocks & $50 / 76(66 \%)$ \\
Head boards & $21 / 34(62 \%)$ \\
Head straps & $36 / 66(55 \%)$ \\
Straps & $83 / 170(49 \%)$ \\
Cervical collars & $27 / 57(47 \%)$ \\
Total & $279 / 491(57 \%)$ \\
\hline
\end{tabular}

Table 2 Ambulance trauma equipment contamination on Kastle-Meyer testing (hospital staff cleaned versus ambulance crew cleaned)

\begin{tabular}{lcc}
\hline & \multicolumn{2}{l}{ Proportion +ve for blood (\%) } \\
\cline { 2 - 3 } Equipment & Hospital staff cleaned & Ambulance crew cleaned \\
\hline Extrication boards & $11 / 16(69 \%)$ & $4 / 8(50 \%)$ \\
Boxsplints & $21 / 28(75 \%)$ & $4 / 11(36 \%)$ \\
Head blocks & $23 / 32(72 \%)$ & $6 / 16(37 \%)$ \\
Head boards & $11 / 16(69 \%)$ & $5 / 8(62 \%)$ \\
Head straps & $20 / 32(62 \%)$ & $7 / 16(44 \%)$ \\
Straps & $41 / 90(46 \%)$ & $21 / 48(44 \%)$ \\
Cervical collars & $13 / 27(48 \%)$ & $5 / 16(31 \%)$ \\
Total & $140 / 241(58 \%)$ & $52 / 123(42 \%)$ \\
\hline
\end{tabular}

Difference in contamination $(95 \% \mathrm{Cl}) 15.8 \%$ (5.0 to $26.6 \%$ ).

10000 solution. It is an indirect test, so is not toxic to the tested surface. False positive results may occur as powerful oxidising agents (for example, copper and iron salts) will turn the Kastle-Meyer solution pink but this will happen before addition of the hydrogen peroxide. Plants contain relatively weak peroxidases which take a long time to induce colour changes in a sample. To avoid this we read the results at five seconds.

Using the Kastle-Meyer technique described, over half of trauma equipment (57\%) tested positive for blood, including $15 \%$ of equipment that was visibly stained with blood. A statistically significant difference was found with respect to blood contamination of equipment between ambulances that retained and cleaned their own equipment and ambulances that left with replacement "clean" equipment from the emergency department storeroom (difference 15.8\% (95\% CI 5.0 to $26.6 \%)$ ). This is unlikely to be clinically significant as $42 \%$ of equipment cleaned by ambulance crews was contaminated.

In ideal conditions the hepatitis B virus can remain viable in dried blood for up to four weeks. ${ }^{5}$ Factors advantageous to viral survival (cold temperatures, lack of ultraviolet light, presence of organic matter ${ }^{67}$ may be present in some

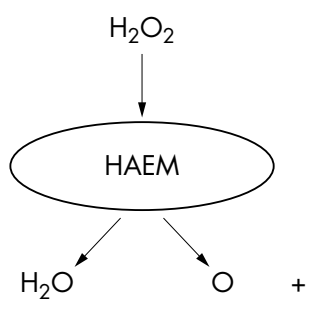

KM

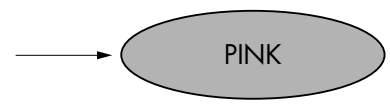

Figure 1 Chemical basis for the colour change of the Kastle-Meyer (KM) solution. ambulances and emergency department store rooms. Although it was not the aim of the study, questioning of healthcare personnel revealed deficiencies in knowledge of Department of Health (DoH) guidelines for decontamination of bloodstained equipment. The practice of washing heavily contaminated equipment by hosing with cold water was prevalent, as was the cleaning of less extensively blood contaminated areas with alcohol impregnated wipes. Cold water may harden fats (hepatitis B and HIV are lipid viruses) and hot water may cause adherence of proteinaceous material. ${ }^{8}$ Alcohol wipes (70\% isopropyl alcohol) kill most bacteria and enveloped viruses but may need to be in contact with the area for at least five minutes. ${ }^{9}$ Due to rapid evaporation, this length of contact cannot be guaranteed by simply wiping the equipment. Sterilisation of equipment that is "in close contact with a break in the skin" is recommended by the Microbiology Advisory Committee to the DoH. For devices with heat labile surfaces, high level chemical disinfection may be used but the DoH stresses that disposable equipment is the preferred option in this situation. ${ }^{11}$ Trauma equipment is usually owned by the regional ambulance service but cleaned and stored in emergency departments and thus may escape standard trust quality assurance programmes.

At any given time, most emergency departments will host a maximum of two extrication boards. If a system of rotation of equipment is adopted then a larger pool of equipment will be necessary to allow thorough decontamination of the equipment by the Central Sterile Services Department. Disposable covers for boards, disposable straps, and disposable radiolucent head restraints are currently commercially available but are seldom used in trauma care and have resource implications. Not all trauma patients have open wounds and many will be immobilised because of "mechanism of injury" concerns over potential cervical spine injury. ${ }^{12}$ It may be possible for ambulance crews to use reusable head restraints, for example, for patients with no wounds and disposable head restraints for trauma cases with bleeding. Reusable equipment would then be classified as low risk ("contact with healthy skin") equipment and could readily be cleaned with alcohol impregnated wipes.

There have been no recorded cases of infection from contaminated trauma equipment but our study has identified the potential risk. Disposable covers for boards, disposable straps, and disposable radiolucent head restraints, which are currently available, provide a solution but have resource implications.

\section{ACKNOWLEDGEMENTS}

The authors would like to thank the clinical directors of the emergency departments and the directors of the ambulance services for their permission to test equipment, and the individual ambulance crews for their cooperation. We would also like to thank the members of the Mid-Yorkshire Research Group and Dr J Brenchley for their helpful comments on the structuring of the paper.

\section{Authors' affiliations \\ J B Lee, A Walker, Pinderfields General Hospital \\ M Levy, The General Infirmary at Leeds, Leeds, UK \\ Funding: funding for the study was provided by the Faculty of Emergency Medicine Research Committee. Results presented at the Faculty of Accident and Emergency Medicine Conference (November 2004). The researchers were independent from the funders. \\ Competing interests: none. \\ Ethics: ethical approval was not required. \\ Correspondence to: Mr J B Lee, Pinderfields General Hospital; docjasonlee@hotmail.com}

Accepted for publication 21 May 2005 


\section{REFERENCES}

1 Edmunds LM, Rawlinson A. The effect of cleaning on blood contamination in the dental surgery following periodontal procedures. Aust Dent $J$ 1998;43:349-53.

2 Anon. Study on blood contamination reveals disturbing results. Synergy News, 2003. Available at http://www.sor.org/members/snnarchive/ SNRAug03p07.pdf (accessed November 2005).

3 Perry SM, Monaghan WP. The prevalence of visible and/or occult blood on anesthesia and monitoring equipment. AANA J 2001:69:44-8.

4 Glaister J. The Kastle-Meyer test for detection of blood. BMJ 1926;10:650-2.

5 Bond WW, Favaro MS, Peterson NJ, et al. Survival of hepatitis B virus after drying and storage for one week. Lancet 1981;1:550-1.

6 Abad FX, Pinto RM, Bosch A. Survival of enteric viruses on environmental fomites. Appl Environ Microbiol 1994;60:3704-10.
7 Druce JD Jardine D Locharnini SA, et al Susceptibility of HIV to inactivation by disinfectants and ultraviolet light. J Hosp Infect 1995;30:167-80.

8 Expert Advisory Group on AIDS and the Advisory Group on Hepatitis. Guidance for clinical health care workers: protection against blood-borne viruses, 1998.

9 Sopwith W, Hart T, Garner P. Preventing infection from reusable medical equipment: a systematic review. BMC Infect Dis 2002;2:4.

10 Department of Health. Decontamination of medical devices, HSC 2000/032, 2000:2.

11 Medical Advisory Committee (MAC). Sterilization, cleaning and disinfection of medical equipment guidance on decontamination to the Department of of medical equipment guidance on decon

12 National Institute for Health and Clinical Excellence. NICE head injury guidelines. Available at http://www.nice.org.uk laccessed November 2005). 


\section{CORRECTIONS}

doi: 10.1136/emj.2005.25346corr l

The author affiliations and correspondence details are incorrect for the paper titled, Use of a forensic technique to identify blood contamination of emergency department and ambulance trauma equipment (Emerg Med J 2006;23:73-5). The correct author affiliations are:

JB Lee, M Levy, Yorkshire Deanery UK

A Walker, Mid-Yorkshire Hospital Trust, UK

Correspondence should be sent to: Miss A Walker, Mid-Yorkshire Hospitals Trust, UK; alison.walker1@midyorks.nhs.uk.

doi: 10.1136/emj.2003.13987corr2

Thrombolyse if no contraindications

An error occurred in figure 3 of the paper titled, Simplifying thrombolysis decisions in patients with left bundle branch block (Emerg Med J 2005;22:617-20). The corrections printed in the November issue (Emerg Med $J$ 2005;22:836) was also incorrect. Please find below the correct figure. The journal apologises for this error.

Patient with chest pain and LBBB

ANY of the following criteria

\begin{tabular}{|c|c|}
\hline $\begin{array}{c}\text { ST elevation > } 1 \text { mm } \\
\text { In leads where QRS } \\
\text { is predominantly } \\
\text { positive }\end{array}$ & $\begin{array}{c}\text { ST elevation > } 5 \mathbf{~ m m} \\
\text { In leads where QRS } \\
\text { is predominantly negative } \\
\text { (leads V1, V2 or V3) }\end{array}$ \\
\hline
\end{tabular}

ST depression > $1 \mathrm{~mm}$

In leads V1, V2, V3 